\title{
The influence of filtered back-projection and iterative reconstruction on partial volume correction in PET
}

\author{
E. Rota Kops' ' B. J. Krause ${ }^{2}$ \\ 'Institut für Medizin (Direktor: Prof. Dr. med. K. Zilles), Forschungszentrum Jülich GmbH, Jülich \\ ${ }^{2}$ Abteilung Nuklearmedizin (Direktor: Prof. Dr. med. H.-W. Müller), Heinrich-Heine- \\ Universität Düsseldorf, Abteilung Nuklearmedizin (Direktor: Prof. Dr. med. S. N. Reske), \\ Radiologie III, Universitätsklinikum Ulm, Deutschland
}

\section{Keywords}

PET, MR imaging, 3-dimensional partial volume correction, reconstruction, filtered back-projection, OSEM, phantom experiments

\section{Summary}

Aim: We assess the influence of the reconstruction algorithms [OS-EM for the iterative one vs. a filtered back-projection in Fourier space (DiFT)] on partial volume correction in PET employing a fully 3D 3-compartment MR based PVcorrection algorithm. The gray matter voxels in the PET image - after removal of the white matter and cerebrospinal fluid contribution - are corrected voxel-by-voxel using the image resolution. Material, methods: Phantom measurements and one healthy human brain FDG study were carried out. For the OSEM reconstruction, a combination of iteration steps and subset numbers (It/Sub) was used, whereby in case of no-convergence the image resolution had to be fitted. The results from the DiFT reconstruction were equivalent to those obtained from the OSEM reconstruction with 10/32 combination for objects with widespread activity concentration. For the sphere phantom, the mean recovery based on the actual values achieved $99.2 \%$ \pm 1.8 for all spheres and all reconstruction modes and It/ sub combinations (except for 2/8). In case of the Hoffman $3 \mathrm{D}$ brain phantom the mean recovery of the cortical regions was $101 \% \pm 1.2$ (the increase based on the uncorrected values: $35.5 \% \pm 1.5$ ), while the subcortical regions reached a mean recovery of $80 \%$ with an increase of $43.9 \% \pm 2.5$. For the human data, an increase of the metabolized values of several cortical regions ranged between $42 \%$ and $48 \%$ independent from the reconstruction mode. Conclusions: Our data show that the 3-compartment fully 3-D MR based PV-correction is sensitive to the choice of reconstruction algorithms and to the parameter choice. They indicate that despite improved spatial resolution, the use of the iterative reconstruction algorithm for PV-correction results in similar recovery factors when compared to a correction using DiFT reconstruction, insofar the image resolution values are fitted at the It/Sub combinations.

\section{Schlüsselwörter}

Positronenemissionstomographie, Kernspintomographie, dreidimensionale Partialvolumen-Korrektur, Rekonstruktion, gefilterte Rückprojektion, OSEM, Phantommessungen

\section{Zusammenfassung}

Ziel: Der Einfluss verschiedener Rekonstruktionsalgorithmen auf die Partialvolumen(PV)-Korrektur von PET-Daten wird untersucht. Material, Methoden: Ein auf MR-Daten basierender dreidimensionaler 3-Kompartment-PV-Korrekturalgorithmus; Phantom-Messungen und eine FDGStudie. Im PET-Bild wurden die Voxel der grauen Substanz mit Hilfe der Bildauflösung nach Entfernung des Beitrages der weißen Substanz und des Liquors korrigiert. Für die OSEM-Rekonstruktion wurde eine Kombination von Iterationsschritten und Anzahl von Subsets (lt/Sub) benutzt. Bei zur Konvergenz unzureichenden Gesamtiterationsschritten wurde die Bildauflösung angepasst. Ergebnisse: Die FBPRekonstruktion war äquivalent zu der OSEM-Rekonstruktion mit der 10/32 Kombination für Objekte mit ausgedehnter Aktivitätsverteilung. Für das Kugelphantom erreichte die mittlere Wiedergewinnung bezogen auf die Sollwerte $99,2 \% \pm 1,8$ für alle Kugeln und It/Sub Kombinationen (außer für 2/8). Beim Hirnphantom war die mittlere Wiedergewinnung der kortikalen Regionen 101\% \pm 1,2 (Anstieg bezogen auf die unkorrigierten Werte: 35,5\% \pm 1,5), wogegen die subkortikalen Regionen eine mittlere Wiedergewinnung von $80 \%$ mit einem Anstieg von 43,9\% $\pm 2,5$ erreichten. Für die ${ }^{18}$ F-FDG-Daten eines menschlichen Gehirns bewegte sich der Anstieg der metabolischen Werte in den kortikalen Regionen zwischen $42 \%$ und 48\%, unabhängig vom Rekonstruktionsmodus. Schlussfolgerung: Unsere Daten belegen, dass der auf MR-Daten basierende dreidimensionale 3-Kompartment-PV-Korrekturalgorithmus empfindlich gegenüber der Wahl des Rekonstruktionsalgorithmus und seiner Parameter ist. Die iterative Rekonstruktion liefert ähnliche Wiedergewinnungsfaktoren im Vergleich zu einer Korrektur nach einer FBP-Rekonstruktion, wenn die Bildauflösungswerte an die It/Sub Kombination angepasst werden.

\section{Der Einfluss der gefilterten Rückprojektion und der itera- tiven Rekonstruktion auf die Partialvolumen-Korrekłur von PET-Daten}

$\mathrm{P}$ ositron emission tomography (PET) allows the quantitative in-vivo measurement of the regional distribution of radioactive tracers. However, the low spatial resolution of PET scanners is a limiting factor for an accurate quantification of, for example, blood flow, metabolism, and receptor density in anatomical structures such as the cerebral cortex. The low spatial resolution results in a partial spread of the structure's signal into the neighbouring tissues for structures which are smaller than twice the resolution of the tomograph $(9,10,12)$, as well as a contribution from these tissues into the considered structure.

As a consequence of this partial volume (PV) effect, the measured PET signal represents a mean radioactivity concentration, which is different from the true radioactivity concentration. In case of cerebral cortex the measured radioactivity concentration is underestimated because the neighbouring compartments, i. e. white matter (WM) and cerebrospinal fluid (CSF) exhibit a lower radioactivity concentration. The $\mathrm{PV}$-effect is affected $(9,10,12)$ by the

- shape of the considered structure,

- surrounding background, and

- axial sampling of the scanner.

After first studies which characterized the PV-effect in brain studies $(2,4,8,23)$, highresolution MR based methods were developed to correct for contributions from CSF $(19,32)$ and to additionally account for WM (24). The latter method was extended including different volumes of interest within the gray matter structures (20). 
A similar idea was presented earlier (28) for a PV-correction based on regional contributions of distinct brain regions incorporating anatomical information into timeactivity data from neuroreceptor PET studies, which was validated with phantom measurements (27). Most of this work contained an error analysis because of the restrictive assumptions about the chosen parameters of the different models that were used. A study dedicated only to such an analysis (31) gives an overview.

Almost all the work on PV-correction algorithm accuracy has been carried out using PET data reconstructed by filtered backprojection algorithms. The use of an iterative reconstruction algorithm can improve spatial resolution (14) and this should subsequently influence the accuracy of PV-correction. However, the frequently used OSEM (Order of Subsets-Expectation Maximization) algorithm is object dependent (25), and represents a confound for the correction. In the present work, the PV-correction is based on the work of MüllerGärtner et al. (24) and our algorithm represents a fully 3-dimensional approach, where also the Gaussian filtering takes into account the third dimension.

The choice of the phantoms was guided by the idea to firstly use a clear and simply arranged phantom (sphere phantom) and in a second step a brain phantom to enable a controlled and comprehensive validation. Phantom experiments were performed in order to provide an error analysis of parameters influencing the performance of the presented three-compartment 3D PV-correction algorithm with the influence of the choice of the reconstruction algorithms (a filtered back-projection in Fourier space (DiFT) (30) vs. iterative reconstruction algorithms (OSEM) being of particular interest.

\section{Methods and materials}

\section{Theory \\ MRF segmentation of MR images}

The Markov random field (MRF) segmentation algorithm used for the segmentation of brain MR images (7) is based on an adap- tive segmentation algorithm published by Wells et al. (35), and incorporates the following important characteristics:

- nonparametric distribution of tissue intensities are described by Parzenwindow statistics,

- neighbourhood tissue correlations are taken into account by a probabilistic approach using MRF,

- signal inhomogeneities are modelled by a a priori MRF.

\section{D PV-correction}

The algorithm of the 3D PV-correction method and the basic equations are briefly presented. The measured 3D PET radioactivity concentration $\mathrm{C}_{\mathrm{PETmeas}}$ is assumed to be the sum of three different true activity concentrations for gray matter $\mathrm{C}_{\mathrm{GM}}$, white matter $\mathrm{C}_{\mathrm{WM}}$, and cerebral spinal fluid $\mathrm{C}_{\mathrm{CSF}}$, which are blurred by the three dimensional image resolution $\mathbf{h}$ :

$$
\begin{aligned}
& \mathrm{C}_{\mathrm{PETmeas}}= \\
& \mathrm{C}_{\mathrm{GM}} \otimes \mathbf{h}(\mathrm{r})+\mathrm{C}_{\mathrm{WM}} \otimes \mathbf{h}(\mathrm{r})+\mathrm{C}_{\mathrm{CSF}} \otimes \mathbf{h}(\mathrm{r})(1)
\end{aligned}
$$

$\mathbf{h}$ depends on the PET system and the reconstruction parameters used resulting in spatially variable values across the scanned volume and $r$ indicates this dependency given the distance from the center of the field of view; the symbol $\otimes$ represents the $3 \mathrm{D}$ convolution. Brain MR data were classified into gray and white matter as well as cerebral spinal fluid using the 3D Markov random field segmentation algorithm (7) shortly described above and the following 3D masks are created:

- gray matter mask $\mathrm{X}_{\mathrm{GM}}$ : the gray matter (GM) voxels are set to 1 , all others to 0 ; - white matter mask $X_{\mathrm{WM}}$ : the white matter (WM) voxels are set to 1 , all others to 0 , - CSF mask $X_{\mathrm{CSF}}$ : the cerebral spinal fluid (CSF) voxels are set to 1 , all others to 0 .

ROIs (regions of interest) should be drawn on the PET images based on the registered and segmented MR image in areas of white matter (e.g. centrum semiovale) and CSF (e.g. ventricles), that are not affected by the PV-effect to obtain the PET radioactivity concentrations $\mathrm{C}^{*}{ }_{\mathrm{WM}}$ for $\mathrm{WM}$ and $\mathrm{C}^{*}{ }_{\mathrm{CSF}}$ for CSF. Using the masks created from the seg- mented data, ideal $3 \mathrm{D}$ radioactivity distributions for white matter and CSF can be calculated:

$$
\mathrm{C}_{\mathrm{WM}}=\mathrm{X}_{\mathrm{WM}} \cdot \mathrm{C}^{*}{ }_{\mathrm{CSF}} \text { and } \mathrm{C}_{\mathrm{CSF}}=\mathrm{X}_{\mathrm{SCF}} \cdot \mathrm{C}^{*}{ }_{\mathrm{CSF}}
$$

Because of the influence of the PV-effect, the corresponding value for $\mathrm{GM}, \mathrm{C}_{\mathrm{GM}}^{*}$, as well as the artificial radioactivity distribution for GM is unknown

$\mathrm{C}_{\mathrm{GM}}=\mathrm{X}_{\mathrm{GM}} \cdot \mathrm{C}^{*}{ }_{\mathrm{GM}}$

Using equation (2), equation (1) can be rewritten to calculate the unknown radioactivity concentration for GM:

$\mathrm{C}_{\mathrm{GM}} \otimes \mathbf{h}(\mathrm{r})=\mathrm{C}_{\mathrm{PET} \text { mean }}-\left(\left(\mathrm{X}_{\mathrm{WM}} \cdot \mathrm{C}^{*}{ }_{\mathrm{WM}}\right) \otimes\right.$
$\left.\mathbf{h}(\mathrm{r})+\left(\mathrm{X}_{\mathrm{CSF}} \cdot \mathrm{C}^{*}{ }_{\mathrm{CSF}}\right) \otimes \mathbf{h}(\mathrm{r})\right)=\mathrm{C}_{\text {uncorr-GM }}$

In this way, the originally measured PET volume is modified by subtracting the contribution of WM and CSF so that only the blurred, uncorrected contribution of GM $\mathrm{C}_{\text {uncorr-GM }}$ is obtained. A homogeneous radioactivity distribution within the GM is assumed, i.e., there are only very small, negligible regional changes of radioactivity distribution, and therefore:

$\mathrm{C}_{\mathrm{GM}} \otimes \mathbf{h}(\mathrm{r})=\left(\mathrm{X}_{\mathrm{GM}} \cdot \mathrm{C}^{*}{ }_{\mathrm{GM}}\right) \otimes \mathbf{h}(\mathrm{r})=$ $\mathrm{C}^{*}{ }_{\mathrm{GM}}\left(\mathrm{X}_{\mathrm{GM}} \otimes \mathrm{h}(\mathrm{r})\right)$

Combining equations (4) and (5) the PVcorrected PET radioactivity concentration for GM is obtained

$\mathrm{C}_{\mathrm{GM}}=\frac{\mathrm{C}_{\mathrm{uncorr}-\mathrm{GM}}}{\left(\mathrm{X}_{\mathrm{GM}} \otimes \mathbf{h}(\mathrm{r})\right)}$

Summarizing at this point, firstly the blurred, uncorrected concentration of GM $\mathrm{C}_{\text {uncorr-GM }}$ is created with the help of segmented MR data set and the measured PET values for WM and CSF. Secondly, these PET data are corrected voxel-by-voxel dividing them with the blurred GM 3D mask obtained with the help of the segmented 3D MR data set. In this way only the GM voxels are considered and corrected. To carry out the convolution operations appearing in equations (4) and (6), a 3D Gaussian filtering was chosen, the shape of which is de- 
scribed in the next section. Furthermore, the non central position of the object in the field of view (FOV) during the PET scan was taken into account.

\section{Spatial resolution measurements}

The resolution values $\mathbf{h}(\mathbf{r})$ used in equations (4) and (6) were determined by measuring line sources in water. Firstly, thin glass pipes $\left(<1 \mathrm{~mm}\right.$ diameter) filled with a ${ }^{18} \mathrm{~F}$-solution were placed parallel to the scanner axis and in different radial positions $(\mathrm{r}=1 \mathrm{~cm}, 4.5$ $\mathrm{cm}, 9 \mathrm{~cm}$ ) to determine the in-plane resolution values, and secondly transversally in the field of view and moved by a step of 1 $\mathrm{mm}$ over $45 \mathrm{~mm}$ to determine the axial resolution.

The data were reconstructed with the standard parameters given in section "Image Processing and Analysis" and the resulting profiles were interpolated linearly. In case of axial resolution profiles were drawn at radial positions of $\mathrm{r}=1 \mathrm{~cm}, 4.5 \mathrm{~cm}$, and $8 \mathrm{~cm}$ on the imaged line source. Our resolution measurements (Fig. 1) are in line with earlier results $(1,5)$ and revealed very similar values for $2 \mathrm{D}$ and $3 \mathrm{D}$.

Dealing with the OSEM data, 6 representative combinations of iteration steps and number of subsets were chosen: $2 / 8,4 / 8$, $4 / 16,6 / 16,10 / 16$, and 10/32 without postfiltering. The combination $6 / 16$ corresponds to the default setting of the scanner Ecat Exact HR+, while 10/32 corresponds to the highest combination allowed from the software which should ensure good convergence and so as good as possible object independent resolution for objects with distributed activity concentration.

The measurements showed that the resolution values were independent from the different combinations of iteration steps and number of subsets. This indicates that nearly full convergence was already achieved for the line sources at 2 iterations and 8 subsets. We decided to chose one representative set of values for the three dimensions and applied interpolated resolution values corresponding to the distances of the interesting regions from the center of the FOV. Same interpolation was done with the DiFT resolution values.

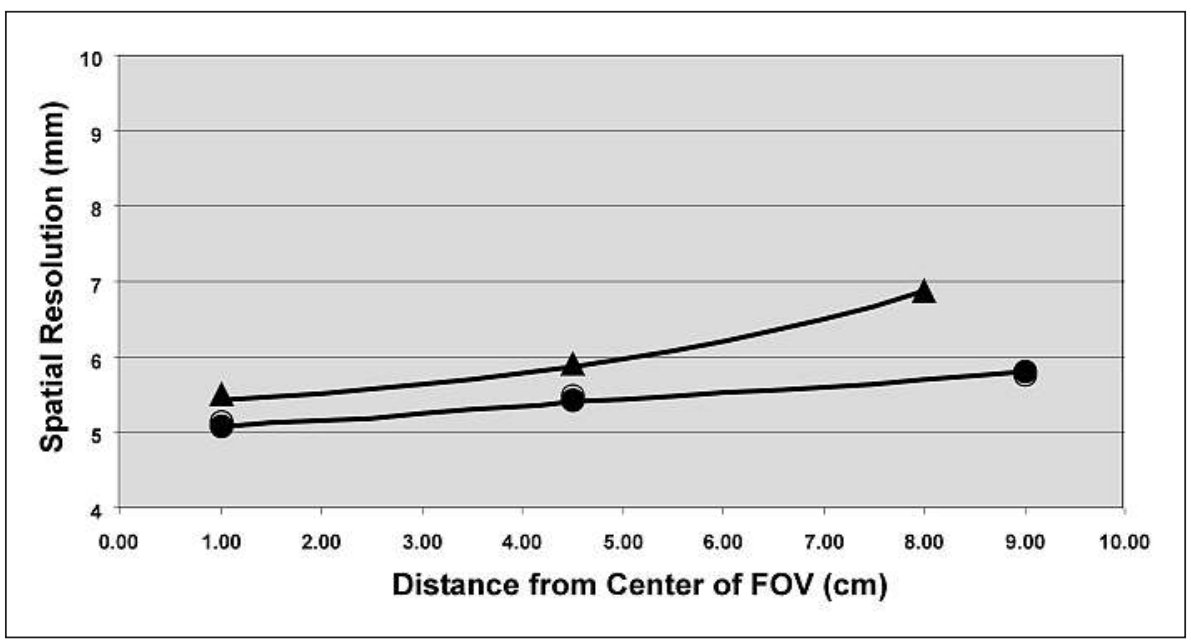

Fig. 1 Mean (2D and 3D) image resolution in case of DiFT as function of the distance from the center of the FOV: radial (O), tangential (O), and axial (A).

\section{Phantom experiments}

Two phantoms were used. The MR measurements were performed with a Magnetom Vision at 1.5 Tesla (Siemens AG, Erlangen, Germany) using a 3D MPRAGE sequence and a voxel volume of $1 \times 1 \times 1 \mathrm{~mm}^{3}$. All PET measurements were carried out with a Siemens Ecat Exact $\mathrm{HR}^{+}$scanner (CTI PET Systems, Knoxville, TN, USA) (1) in both 2D and 3D modus. For all experiments, a cold transmission scan was performed for measured attenuation correction. In order to obtain the true radioactivity concentrations for the different phantom measurements, a well counter was used as a reference.

\section{Cylindrical phantom}

A cylindrical phantom $(20 \mathrm{~cm}$ diameter $)$ containing six glass spheres (wall thickness: about $1 \mathrm{~mm}$ ) with different diameters was used (PTW Freiburg, Germany) (6). The spheres were fixed in the cylinder so that the maximum diameters were positioned at the same level. The diameters ranged from 9.6 $\mathrm{mm}$ to $35.6 \mathrm{~mm}$ (Fig. 2a).

For PET measurements the ${ }^{18} \mathrm{~F}$ radioactivity concentration of the spheres was $36.8 \mathrm{kBq} / \mathrm{cc}$, while the cylinder had a background concentration of $6.9 \mathrm{kBq} / \mathrm{cc}$. The 2D scan was first performed and was followed by the 3D scan about $25 \mathrm{~min}$ later. The radioactivity concentrations for this latter measurement were $32.0 \mathrm{kBq} / \mathrm{cc}$ in the spheres and $5.9 \mathrm{kBq} / \mathrm{cc}$ in the background. For the MR measurements the spheres as well as the surrounding volume were filled with a solution of nickel sulphate and a concentration ratio of $1: 0.5$.
Fig. 2

Sphere phantom a) six spheres (diameters: $9.6-35.6 \mathrm{~mm}$ ) positioned equatorially; b) corresponding $P E T$ image measured in $2 \mathrm{D}$ and reconstructed with OSEM $10 / 32$
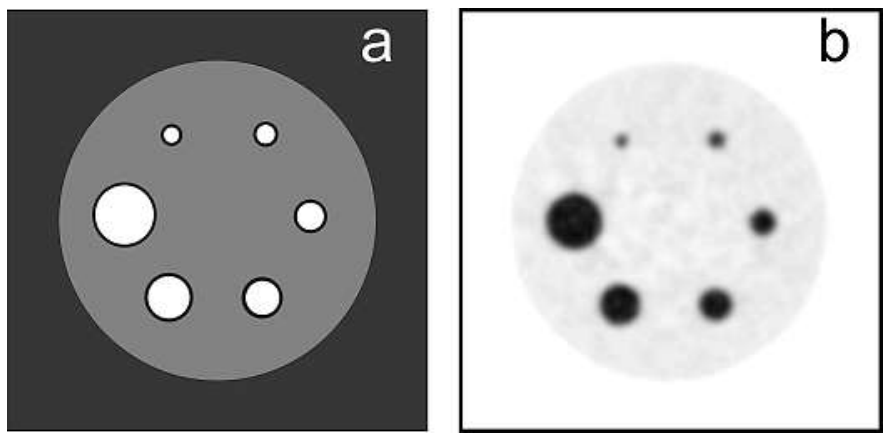


\section{Brain phantom}

The Hoffman 3D brain phantom provides a three dimensional simulation of the radioisotope distribution found in the normal brain (Fig. 3a). It is made of sturdy plastic and a single fillable chamber simulates a 4 : 1 uptake ratio for GM : WM. The dimensions are $17.5 \mathrm{~cm} \times 20.8 \mathrm{~cm}$ and the fillable volume is about $1200 \mathrm{ml}$.

For PET measurements a ${ }^{18} \mathrm{~F}$ solution with $39.7 \mathrm{kBq} / \mathrm{cc}$ was used starting with the 2D scan (WM value: $9.9 \mathrm{kBq} / \mathrm{cc}$ ). After about 1.5 hours the 3D scan was started resulting in a $\mathrm{GM}$ concentration of $22.3 \mathrm{kBq} /$ cc (WM value: $5.6 \mathrm{kBq} / \mathrm{cc}$ ). Instead of a MR scan, a digitized 3D data set of the phantom was used to generate simulated segmented data.
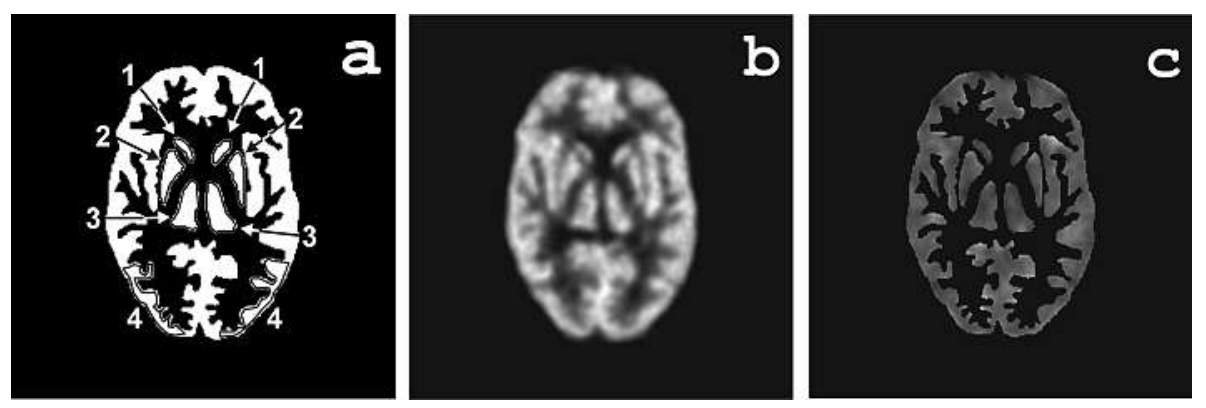

Fig. 3 Hoffman 3D brain phantom simulates a $4: 1$ uptake ratio in the gray and white matter a) ROIs corresponding to (1) caudate nucleus, (2) putamen, (3) thalamus, and (4) occipital cortex; b) 3D PET image reconstructed with OSEM 10/32; c) PV corrected
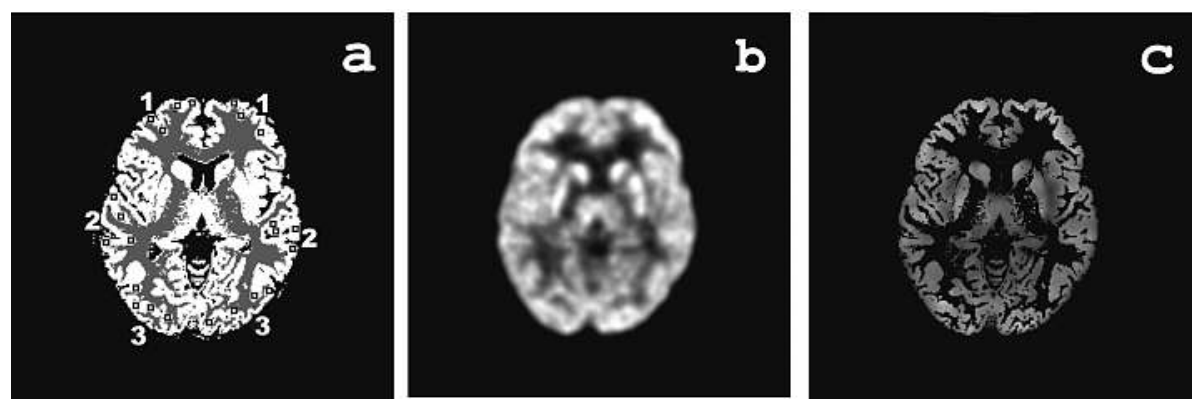

Fig. 4 Human study

a) ROIs corresponding to (1) frontal cortex, (2) parietal cortex, and (3) occipital cortex. The ROIs for the temporal cortex were drawn on another slice. b) 3D PET image reconstructed with OSEM 10/32; c) PV corrected

\section{Image processing and analysis}

All PET data were reconstructed with filtered back-projection in Fourier space as well as with the iterative weighted OSEM algorithm $(11,21)$. Fourier rebinning was used prior to both reconstruction algorithms in case of 3D scan mode. Data were corrected for scatter, random, and attenuation, the latter using measured attenuation values. In case of DiFT, the 63 transaxial images were obtained using a Shepp filter with a cut-off frequency of 0.40 cycles/pixel; in case of OSEM, representative combinations of iteration steps and number of subsets were chosen, as given in section „Spatial Resolution Measurements". The zoom factor was 2.53 and the image matrix $128 \times 128$ yielding a voxel size of $2.035 \times 2.035 \times$ $2.425 \mathrm{~mm}^{3}$.

The MPITool (ATV GmbH, Germany) (26) was used for all data analysis (phantom and human data). After co-registration of the reconstructed PET images with the MR images, the phantom and the human data were transformed into the MR space. The Hoffman phantom had the same in-plane pixel size of $131 \mathrm{~mm}^{2}$, but only 64 slices and a voxel thickness of $2.33 \mathrm{~mm}$ as the digitized image features.

For the phantoms, a recovery factor (expressed as $\%$ of change) was used as a measure of the correction accuracy. It is the ratio between the measured PV uncorrected or corrected radioactivity concentrations $\mathrm{C}_{\text {meas }}$ and the corresponding true concentration values $\mathrm{C}_{\mathrm{GM}}$.

Recovery factor $=100 \cdot \mathrm{C}_{\text {meas }} / \mathrm{C}_{\mathrm{GM}}$

To get $\mathrm{C}_{\text {meas }}$, ROIs were used. On the spheres we placed circles with a diameter of $75 \%$ of the actual diameter in order to assess the influence of the 3D PV-correction by covering most of the surface, but reducing the influence of the borders. For the Hoffman phantom ROIs were placed within two cortical and three subcortical regions following the structure of the corresponding digitized image (Fig. 3a). The calculation of recovery factors was then performed as stated above by dividing the mean activity concentration values from the ROIs by the known values $\mathrm{C}_{\mathrm{GM}}$. For the human data, four 
cortical regions were chosen (Fig. 4a): frontal cortex (1), parietal (2), occipital (3), and temporal (not shown because on a higher cut)) and four rectangular ROIs (size: $3.5 \times 3.5 \mathrm{~mm}^{2}$ ) were drawn on each of them. The mean of these four ROIs was used for the calculation of the increase (\%) subsequent to the PV-correction.

As already mentioned, the resolution values achieved for the OSEM reconstruction are apparently valid for all the iterations and subsets combination. This is in fact not applicable to distributed activity concentrations as the sphere phantom, the Hoffman phantom, and even more for brain images. The commonly used iteration and subset combinations (e.g., 6/16) do not fully converge. The resolution values have consequently to be incremented in order to obtain the real recovery coefficient using the partial volume correction algorithm. We decided to enhance the resolution values stepwise (e.g., $3 \%, 5 \%, 8 \%$ ) and to stop when the recovery factor was nearly $100 \%$. The values achieved for the DiFT reconstruction have been slightly increased, too, in accordance to the increment used for the combination 10/32. For the human data we finally used the increment obtained by the analysis of the Hoffman brain phantom.

\section{Results}

\section{Phantom data}

Figure $2 \mathrm{~b}$ shows a reconstructed image of the sphere phantom scanned in 2D mode and reconstructed with OSEM (10/32). Figure 5 shows the resulting plots of the recovery factors for the DiFT reconstruction (means between 2D and 3D due to the high similarity of the values) (Fig. 5a) and for the OSEM 10/32 reconstruction (Fig. 5b) together with the absolute percentage increase of the uncorrected data after PV-correction. Table 1 shows the percentages of resolution increment corresponding to each iteration/ subset combination. The values of the $2 \mathrm{D}$ and 3D scan modes were almost identical, so one list only is given.

It was possible to reach a very high recovery $(99.2 \% \pm 1.8)$ for all the spheres and

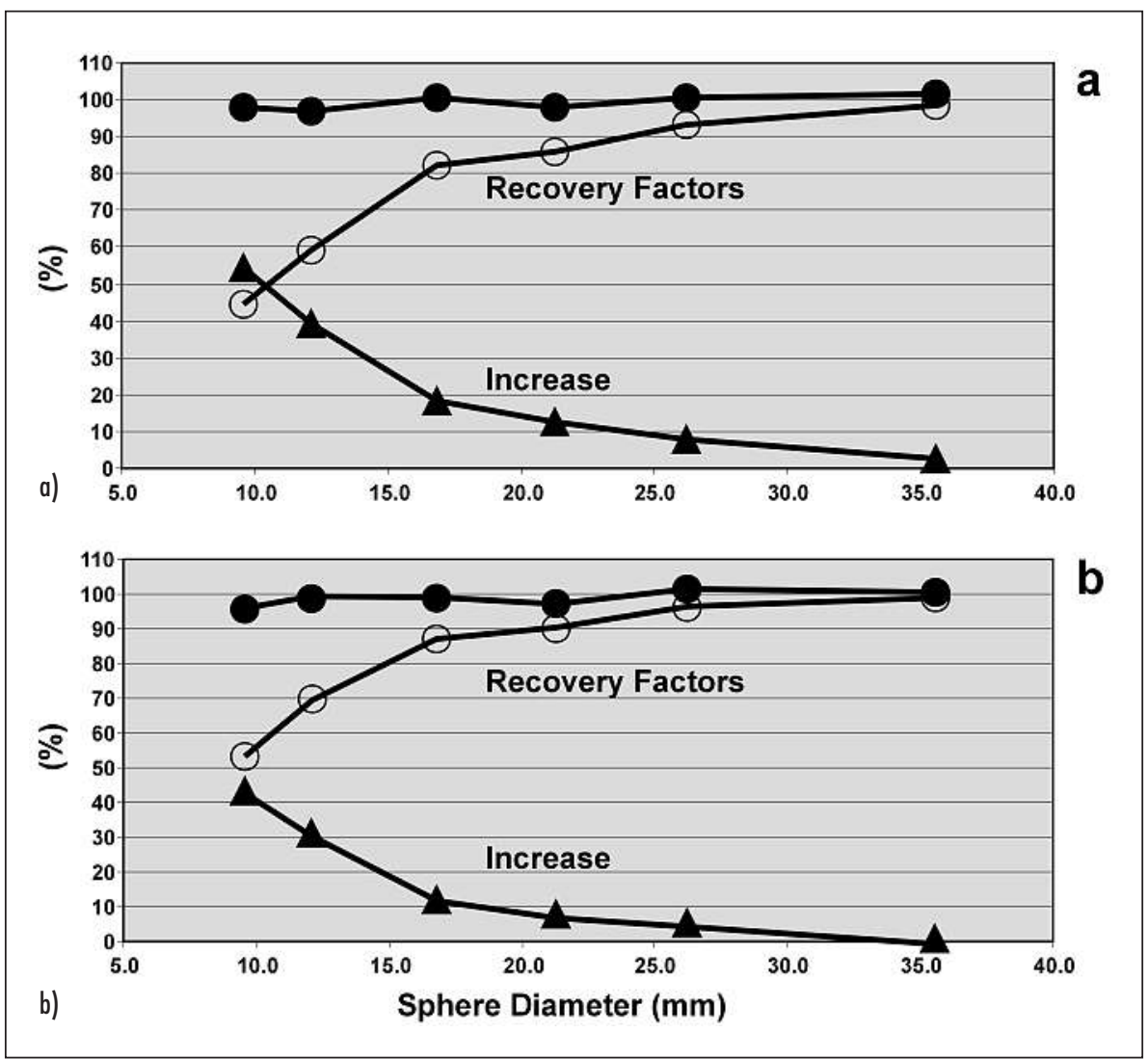

Fig. 5 Sphere phantom: mean (2D and 3D) recovery factors (\%) as function of the sphere diameters for DiFT (a) and OSEM 10/32 (b) reconstructions; uncorrected (O) and PV corrected (O) data plotted against the corresponding percentage increase (A) all the iteration/subset combination, except for the lowest combination of $2 / 8$ even with a increase of $40 \%$, whereby particularly the two smallest spheres could not be corrected enough.

Figure $3 \mathrm{~b}$ shows the uncorrected Hoffman brain phantom scanned in 3D and reconstructed with DiFT, the corresponding $\mathrm{PV}$-corrected image is given in Figure 3c. In Figure 6a the recovery factors for the cortex of the Hoffman 3D brain phantom are shown as vertical bars as function of the single reconstruction types and the percentages of the resolution increment used. The white bar corresponds to the uncorrected data, the gray one to the corrected data without resolution increment, and the black one corresponds to the corrected data with inmean values for $2 \mathrm{D}$ and $3 \mathrm{D}$ data due to the very similar results. In the same way, Figure $6 \mathrm{~b}$ shows the recovery factors for the caudate nucleus which represents the region with the poorest recovery.

The stop criterion for the resolution increment in the case of the Hoffman brain phantom was chosen on the basis of the cortex values, so that for the cortex regions the cremented resolution values. These are

Tab. 1 Sphere phantom data: overestimation (\%) of the resolution values for the different iteration/subset combinations of the OSEM and the DiFT reconstruction listed together with the mean percentage increase of the uncorrected values after PVcorrection

\begin{tabular}{|lccccccc|}
\hline 2D \& 3D & DiFT & OS-EM & OS-EM & OS-EM & OS-EM & OS-EM OS-EM & OS/10/16 \\
& & $10 / 32$ & $10 / 16$ & $6 / 16$ & $4 / 16$ & $4 / 8$ & $2 / 8$ \\
$\begin{array}{l}\text { resolution } \\
\text { increment (\%) }\end{array}$ & $+10 \%$ & $+3 \%$ & $+10 \%$ & $+10 \%$ & $+12 \%$ & $+16 \%$ & $+40 \%$ \\
\hline
\end{tabular}



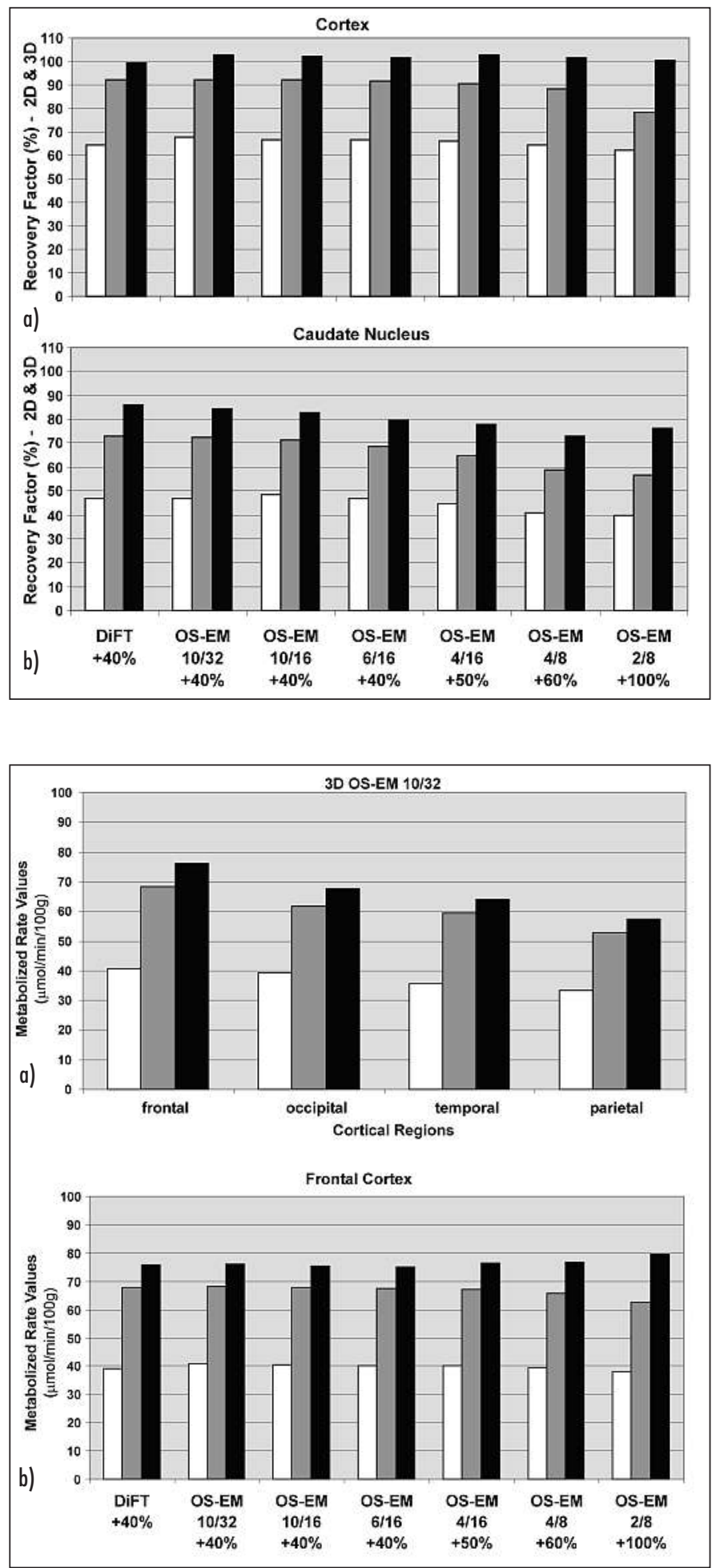

Fig. 6

Hoffman 3D brain phantom: mean (2D and $3 D$ ) recovery factors for the cortex (a) and for the caudate nucleus (b) as function of the reconstruction modes. The overestimation used for the resolution values is indicated. white bars: uncorrected data; gray bars: PV-corrected data without resolution overestimation; black bars: PV-corrected data with overestimation

Fig. 7

Metabolic rate values of the human study obtained with OSEM 10/32 (overestimation for resolution values indicated); white bars: uncorrected data; gray bars: PV-corrected data without resolution overestimation; black bars: PV-corrected data with overestimation a) as function of the cortical regions; b) frontal cortex as function of the reconstruction modes

recovery reached a mean value over all the reconstruction types of $101 \%$ and a mean percentage increase from the uncorrected data of $35.5 \% \pm 1.5$. Using these incre-

\section{Human brain data}

Figure $4 \mathrm{~b}$ shows the uncorrected metabolic rate values scanned in $3 \mathrm{D}$ and reconstructed with DiFT; Figure 4c shows the corresponding PV-corrected image. In Figure 7a the metabolic values obtained with OSEM $10 / 32$ of the human data are shown as vertical bars for the different cortical regions. Figure $7 \mathrm{~b}$ shows the metabolic values for the frontal cortex as function of the reconstruction modes. Again, the white bar corresponds to the uncorrected data, the gray one to the corrected data without increment of the resolution values, and the black one corresponds to the corrected data with incremented resolution values. The considered increment was the same as used for the Hoffman 3D brain phantom and the values are indicated in figure $7 \mathrm{~b}$, too.

The mean values of the cortical areas, calculated as the mean over four rectangular ROIs in each area, showed a clear dependency on the location in the brain. Within each area the percentage increase based on the uncorrected data and due to the PV-correction was rather constant over all the reconstruction modes, ranging from $36 \%$ up to $42 \%$ in case of PV-correction with no incremented resolution values, while ranging from $42 \%$ up to $48 \%$ when the resolution increment was taken into account.

\section{Discussion}

The PV-correction algorithm presented here is based on the work of Müller Gärtner et al. (24) and represents a fully 3-dimensional approach. Specifically, we have addressed the question as to whether and if so to which extent PET data reconstruction influences the performance of the 3D PV-correction algorithm. Until now almost all of the work on PV-correction methods made use of filtered back-projection in the framework of PET data reconstruction $(13,16-18,20)$. For the first time, an iterative reconstruction algorithm was used and comparisons on the potential influence of the choice of the PET image reconstruction algorithms on the quality of a PV-correction algorithm are presented. 
In a first step the PET data sets (corrected for scatter, random and attenuation) were reconstructed with a filtered back-projection algorithm in Fourier space (DiFT) (30). Our findings using the DiFT algorithm are generally in line with findings of other groups that used filtered back-projection for data reconstruction. The comparison of real phantom data shows that the recovery factors for reconstructed real data that also contain noise - as compared to simulated data does not always reach $100 \%$ and is lower for small structures like in this case the two smallest spheres of the phantom. This is in accordance with Strul et al. (31) who pointed out that correction instability is greater for small structures, and segmentation is the most critical factor contributing to the imprecision of correction. We showed that an increment of the optimal resolution values the possible bias due to the noise can be compensated, because the PV-correction strongly depends on the image resolution values used.

To date, PET data reconstruction in the framework of PV-correction algorithm evaluation is performed primarily using filtered back-projection. However, it is known for data with poor statistics, such as PET data, that the filtered back-projection results in low signal-to-noise ratio and streak artifacts. Iterative reconstruction algorithms have been introduced $(3,15,22,29,33,34)$ that show an improved image contrast and signal-to-noise ratio (22) as well as an improved resolution. Possible confounding factors of OSEM are related to slow convergence, amplification of noise with increasing number of iterations and dependence of its characteristics on source distribution. Furthermore, OSEM produces images with varying spatial resolution that is dependent on the surrounding activity. Defining criteria for stopping the iteration before the image becomes too noisy is very difficult since different parts of the image converge at different rates leading to non-uniform and object dependent resolution. Thus, the OSEM has position dependent (and image dependent) bias and resolution. For some applications this position dependent bias and resolution may play an important role. E.g., in tracer kinetic modeling, the time activity curves should only reflect changes in tracer concentration, and changes due to varying spatial resolution will cause errors. Similarly, when applying a PV-correction it is important that the bias does not change with position and image contents.

In order to assess the influence of the choice of the reconstruction algorithm on the PV-correction, the PET data sets (again corrected for scatter, random and attenuation) in a second step were reconstructed with an iterative reconstruction algorithm, OSEM $(11,21)$. Using the OSEM algorithm, the recovery factor depends not only on the image resolution, but also on the It/ Sub combinations, i.e., on the achieved convergence: The use of the iterative OSEM algorithm with high It/Sub combinations (e.g., 10/32) resulted in similar recovery factors when compared to the filtered backprojection algorithm. At lower It/Sub combinations the image resolution values, obtained from a line source which can be imaged already at very low It/Sub combinations due to the simple object form, have to be incremented in order to reach a fully recovery and eventually compensate for the bias introduced by the noise. Because the shape of the sphere phantom is geometrically "simpler" than that of the Hoffman phantom with distributed activity concentration, we observed that the percentage increments for these two kinds of phantoms turned out to be different. In the case of the Hoffman phantom small structures like the caudate nucleus showed a reduced recovery factor although the cortex could be recovered up to $100 \%$. At present we do not have an explanation of this finding, which should be further examined, also taking into account possible image reconstruction and correction biases. For the brain study we applied the same increments of the resolution values as for the Hoffmann phantom to obtain images with sufficient accuracy.

It can be concluded that for larger structures OSEM and DiFT provide similar results, given that the It/Sub combinations provide high convergence. Optimization of the number of iterations is, however, required for each organ scanned, as previously shown by Yao et al. (36) as well as the optimization of the image resolution increment depending on the It/Sub combination.

\section{Conclusion}

The correction of partial volume effects represents an important step towards the improvement of PET quantification especially with respect to PET studies on ageing or neuro degenerative disease. We presented a fully 3D 3-compartment PV-correction algorithm and applied it to simplified models and phantom shapes as well as to a single human brain study to show globally its influence. As an important finding we demonstrate that the accuracy of the 3D PV-correction algorithm depends on the used reconstruction algorithms (filtered back-projection and iterative reconstruction): OSEM revealed still an overall higher sensitivity than the DiFT and the effect was most prominent at lower It/Sub combinations being in line with the idea that it is a resolution problem, due to the difficulty to obtain resolution values independent from object shape and radioactivity distribution.

\section{References}

1. Adam LE, Zaers J, Ostertag H et al. Performance evaluation of the whole-body PET scanner ECAT Exact HR+ following the IEC standard. IEEE Trans Nucl Sci 1997; 44: 1172-9.

2. Bendriem B, Dewey SL, Schlyer DJ et al. Quantitation of the human basal ganglia with positron emission tomography: a phantom study of the effect of contrast and axial positioning. IEEE Trans Med Imag 1991; 10: 216-21.

3. Comtat C, Kinahan PE, Defrise $M$ et al. Fast reconstruction of 3D PET data with accurate statistical modeling. IEEE Trans Nucl Sci 1998; 45: 1083-9.

4. Cooke BE, Evans AC. A phantom to assess quantitative recovery of positron tomographs. J Comput Assist Tomogr 1983; 5: 876-80.

5. ECAT Software Manual.

6. Guzzardi R, Bellina C, Knoop B et al. Methodologies for performance evaluation of positron emission tomographs. J Nucl Biol Med 1991; 35 : 141-57.

7. Held K, Rota Kops E, Krause BJ et al. Markov random field segmentation of brain MR images. IEEE Trans Med Imag 1997; 16: 878-86.

8. Hoffman EJ, Cutler PD, Guerrero TM et al. Assessment of accuracy of PET utilizing a 3-D phantom to simulate the activity distribution of ${ }^{18} \mathrm{~F}$-fluorodeoxyglucose uptake in the human brain. J Cereb Blood Flow Metab 1991; 11 : A17-25.

9. Hoffmann EJ, Huang SC, Phelps ME. Quantitation in positron emission tomography: 1. Effect 
of object size. J Comput Assist Tomogr 1979; 3 : 299-308.

10. Hoffmann EJ, Huang SC, Plummer D et al. Quantitation in positron emission computed tomography: 6. Effect of nonuniform resolution. J Comput Assist Tomogr 1982; 5: 987-99.

11. Hudson HM, Larkin RS. Accelerated image reconstruction using ordered subsets of projection data. IEEE Trans Med Imag 1994; 13: 601-9.

12. Kessler RM, Ellis JR, Eden M. Analysis of emission tomographic scan data: limitations imposed by resolution and background. J Comput Assist Tomogr 1984; 3: 514-22.

13. Labbè C, Froment JC, Kennedy A et al. Positron emission tomography metabolic data corrected for cortical atrophy using magnetic resonace imaging. Alzheimer Dis Assoc Disord. Fall 1996; 10: $141-70$.

14. Liow JS, Strother SC, Rehm K et al. Improved resolution for PET volume imaging through three-dimensional iterative reconstruction. J Nucl Med 1997; 38: 1623-31.

15. Lipinski B, Herzog H, Rota Kops E et al. Expectation maximization reconstruction of positron emission tomography images using anatomical magnetic resonance information. IEEE Trans Med Imag 1997; 16: 129-36.

16. Meltzer CC, Bryan RN, Holcomb HH et al. A method of anatomical localization for positron emission tomography using magnetic resonance imaging. J Comput Assist Tomogr 1990; 14: $418-26$.

17. Meltzer CC, Cantwell MN, Greer PJ et al. Does cerebral blood flow decline in healthy aging? A PET study with partial-volume correction. J Nucl Med 2000; 41: 1842-48.

18. Meltzer CC, Kinahan PE, Greer PJ et al. Comparative evaluation of MR-based partial volume correction schemes for PET. J Nucl Med 1999; 40: 2053-65.

19. Meltzer CC, Leal JP, Mayberg HS et al. Correction of PET data for partial volume effects in human cerebral cortex by MR imaging. J Comput Assist Tomogr 1990; 14: 561-70.

20. Meltzer CC, Zubieta JK, Links JM et al. MRbased correction of brain PET measurements for heterogeneous gray matter radioactivity distribution. J Cereb Blood Flow Metab 1996; 16: 650-8.

21. Michel C, Sibomana M, Bol A et al. Preserving Poisson characteristics of PET data with weighted OS-EM reconstruction. Conf. Record of the IEEE Nuclear Science Symposium and Medical Imaging Conference, Toronto 1998.

22. Miller TR, Wallis JW. Clinically important characteristics of maximum-likelihood reconstruction. J Nucl Med 1992; 33: 1678-84.

23. Mullani NA. A phantom for quantitation of partial volume effects in ECT. IEEE Trans Nucl Sci 1989; 36: $983-7$.

24. Müller-Gärtner HW, Links JM, Prince JL et al Measurement of radiotracer concentration in brain gray matter using positron emission tomography: MR-based correction for partial volume effects. J Cereb Blood Flow Metab 1992; 12 : $571-83$.

25. Mustafovic S, Thielemans K, Hogg D et al. Object dependency of resolution and convergence rate in OSEM with filtering. Conference Record IEEE NSS\&MIC, Nov. 2001, San Diego, CA, USA.

26. Pietrzyk U, Herholz K, Fink G et al. An interactive technique for three-dimensional image registration: Validation for PET, SPECT, MRI and CT brain studies. J Nucl Med 1994; 35: 2011-8.

27. Rousset OG, Ma Y, Evans AC. Correction of partial volume effects in PET: principle and validation. J Nucl Med 1998; 39: 904-11.

28. Rousset OG, Ma Y, Léger GC et al. Correction for partial volume effects in PET using MRI-based 3-D simulations of individual human brain metabolism. In: Uemura K, Lassen NA, Jones T et al. (eds). Quantification of brain function tracer kinetics and image analysis in brain PET. Amsterdam: Elsevier Science 1993; 113-25.
29. Schmidlin P, Doll J. Implementation of iterative reconstruction in positron emission tomography. Prog Clin Biol Res 1991; 363: 59-67.

30. Steams CW, Chosler DA, Brownell GL. Three-dimensional image reconstruction in the Fourier domain. IEEE Trans Nucl Sci 1987; NS-34: 374-8.

31. Strul D, Bendriem B. Robustness of anatomically guided pixel-by-pixel algorithms for partial volume effect correction in positron emission tomography. J Cereb Blood Flow Metab 1999; 19: $547-59$.

32. Videen TO, Perlmutter JS, Mintun MA et al. Regional correction of positron emission tomography data for the effects of cerebral atrophy. J Cereb Blood Flow Metab 1988; 8: 662-70.

33. Wang CX, Snyder WE, Bilbro G et al. Performance evaluation of filtered backprojection reconstruction and iterative reconstruction methods for PET images. Comput Biol Med 1998; 28: 13-24.

34. Wang W, Gindi G. Noise analysis of MAP-EM algorithms for emission tomography. Phys Med Biol 1997; 42: 2215-32.

35. Wells III WM, Grimson WEL, Kikinis R et al. Adaptive segmentation of MRI data. IEEE Trans Med Imag 1996; 15: 429-42.

36. Yao R, Seidel J, Johnson CA et al. Performance characteristics of the 3-D OSEM algorithm in the reconstruction of small animal PET images. IEEE Trans Med Imaging 2000; 19: 798-804.

Correspondence to:

Dr. Elena Rota Kops

Institute of Medicine

Forschungszentrum Jülich

52425 Jülich, Germany

Tel. +49/(0)24 61/61-5738, Fax -20 89

E-mail:e.rota.kops@fz-juelich.de 